\title{
Highlights aus den letzten 20 Jahren
}

Wie Sie sicherlich schon über einen Blick auf die Titelseiten des Jahrgangs 2017 bemerkt haben, hat die Dialyse aktuell dieses Jahr einen runden Geburtstag. Es ist nun tatsächlich 20 Jahre her, seit die erste Ausgabe im Mai 1997 erschienen ist - damals noch im Conzema-Verlag, mit einem anderen inhaltlichen Konzept und in einem anders gestalteten Layout.

Wir freuen uns sehr, dass wir über die Jahre viele neue Kooperationspartner und Kunden gewinnen konnten: Sie sind uns treu erhalten geblieben und wir arbeiten sehr erfolgreich zusammen. Und natürlich danken wir unseren Autoren, Gasteditoren und wissenschaftlichen Beiräten herzlich für ihren tatkräftigen Input zur Gestaltung der Inhalte der Zeitschrift, die wir für Sie - unsere Leser - produzieren. Um einmal einen Überblick über die Entwicklung der Dialyse aktuell zu erhalten, finden Sie im Folgenden eine Auswahl an Highlights aus den letzten 20 Jahren - wir hoffen, dass wir diesen in Zukunft noch viele weitere Höhepunkte hinzufügen können!

\section{Auswahl an Highlights in der Dialyse aktuell}

- 1997: Gründung der Zeitschrift durch die Conzema AG, Lohfelden.

- 2000: Ausweitung der Erscheinungsweise von 4 auf 6 Ausgaben pro Jahr.

- 2001: Ausweitung der Erscheinungsweise von 6 auf 7 Ausgaben pro Jahr.

- 2002: Ausweitung der Erscheinungsweise von 7 auf 8 Ausgaben pro Jahr.

- 2004: Im Januar übernimmt der Georg Thieme Verlag die Dialyse aktuell und setzt die Kooperation der Zeitschrift mit der Fachgesellschaft AfnP e. V. fort.

- 2005: Anpassungen im Layout.

- 2006: Beginn der Kooperation mit der Fachgesellschaft AKTX-Pflege e. V.

- 2006: Beginn der Kooperation mit der Fachgesellschaft fnb e. V.

- 2006: Die Dialyse aktuell wird das ausschließliche deutschsprachige Organ der AfnP e. V. und stärkt somit die Bindung zur Fachgesellschaft.

- 2006: Die AfnP e. V. und die Dialyse aktuell vergeben ab 2006 jährlich gemeinsam den Förderpreis Nephrologische Pflege, wobei der Georg

Thieme Verlag als Sponsor für das Preisgeld von 1500 Euro auftritt.

- 2006: Der Georg Thieme Verlag produziert erstmals das Programmheft zum AfnP-Symposium.

- 2007: Umstrukturierung des Layouts.

- 2008: Für den regelmäßigen Bezug der Dialyse aktuell über z. B. ein Abonnement erhalten Pflegekräfte jährlich 3 Punkte der RbP - Registrierung beruflich Pflegender $\mathrm{GmbH}$.

- 2008: Sonderdruck „Dialyseshunts: Fakten - Fragen - Probleme“ aus den ersten 50 Beiträgen zur Rubrik „Shuntecke“ aus der Dialyse aktuell erscheint.

- 2009: Etablierung von Themenschwerpunkten in den einzelnen Ausgaben.

- 2009: Ausweitung der Erscheinungsweise von 8 auf 10 Ausgaben pro Jahr.

- 2010: Das neu entwickelte, jährliche redaktionelle Supplement „Im Fokus“ erscheint erstmals.

- 2011: Der Georg Thieme Verlag wird über die Dialyse aktuell Kooperationspartner sowie Sponsor der jährlichen Nephro Fachtagung Ulm und produziert erstmals das zugehörige Programmheft.

- 2011: Buchausgabe „Dialyseshunts: Fakten - Fragen - Probleme“ aus den ersten 75 Beiträgen zur Rubrik „Shuntecke“ aus der Dialyse aktuell, die aktualisiert wurden, erscheint.

- 2012: Beginn der Kooperation mit der Fachgesellschaft BANP.

- 2013: Installation eines wissenschaftlichen Beirats.

- 2013: CME-Zertifizierung von

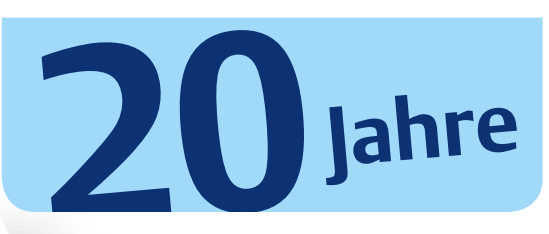

.

Dialyse Aluter
Netern
$=$

Schwer-

punktbeiträgen.

- 2014: Neue Rubrik „Journal-Club Pflege“ erscheint erstmals.

- 2014: Beginn der Kooperation mit der Fachgesellschaft DGfN.

- 2014/2015: Erweiterung des wissenschaftlichen Beirats.

- 2015: Neue Rubrik „Expertentipp“ erscheint erstmals.

- 2015: Beginn der Kooperation mit der Fachgesellschaft EDTNA/ERCA.

- 2015: Buchausgabe „Dialyseshunts: Fakten - Fragen - Probleme“ aus den ersten 100 Beiträgen zur Rubrik „Shuntecke“ aus der Dialyse aktuell, die inhaltlich und redaktionell überarbeitet wurden, plus zusätzliche Artikel zur Klinik von Dialyseshunts plus pflegerische Beiträge erscheint.

- 2016: Neue Rubrik „Showroom“ erscheint erstmals in der Dialyse aktuell.

- 2017: Umstrukturierung des Layouts.

- 2017: Beginn der Kooperation mit der Fachgesellschaft DN e. V.

- 2017: Neue Rubrik „Apherese A-Z“ wird in der zweiten Jahreshälfte eingeführt.

Für das Dialyse aktuell Team Christian Schäfer, Stuttgart 